\title{
A Study of tinea pedis and tinea unguium in patients with dementia
}

Purpose: We conducted a study on clinical and mycological features of tinea pedis and tinea unguium in patients with dementia.

\section{Methods:}

We studied patients with dementia who attended department of neurological medicine of Juntendo University Koshigaya Hospital. All individuals gave informed consent prior to participating in our study. The Ethics Committee of Juntendo School of Medicine approved this study. We examined specimens from sites of possible dermatophyte infection in feet and toenails of the patients by direct microscopy in potassium hydroxide $(\mathrm{KOH})$. When fungus elements were pssitive ( $\mathrm{KOH}$-positive), causative species were identified by culturing the funji on Sabraud's glucose agar. And we also recorded clinical features and estimated severity of tinea pedis (Table 1 ).

\section{Results:}

We studied 64 patients (25 men, 39 women, a mean age 76.8 years). 56 patients had Alzheimer's disease, 3 had dementia with Lewy bodies, 3 had frontal temporal lobar degeneration and others. Of these 64 patients, 39 patients (60.9\%, 15 men, 24 women) presented positive signs by direct microscopy. 20 patients with tinea pedis (51.3\%, 9 men, 11 women) also had tinea unguium.14 patients (35.9\%, 6 men, 8 women) had only tinea pedis, and 5 female patients (20.8\%) had only tinea unguium., The most common clinical type of tinea pedis was the interdigital type and those of tinea unguium was the distal-lateral subungal onychomycosis (DLSO). The mean severity score of tinea pedis was 3.9. Positive cultures were observed in 22 patients (56.4\%). Trichophyton rubrum was isolated in 19 (48.7\%), T. mentagrophytes in $3(7.7 \%)$ (Table 2). No statistically significant differences in tinea pedis and/or tinea unguium incidence rates were observed between male and female (Fisher's exact test).

\section{Discussion:}

Tinea pedis and tinea unguium are the most common dermatophytosis seen in the daily practice of dermatology. According to the report in JAPAN FOOT WEEK 2006, it is estimated that about 1 in 5 Japanese have tinea pedis and abou for 25t 1 in 10 Japanese have tinea unguium According to the same report, $49 \%$ of the foot diseases of new dermatological outpatients were tinea pedis and/or tinea unguium. Another study indicated that patients with occult athlete's foot account for $25 \%$ of all new dermatological outpatients in Japan.
In our study, 70's and 80's of patients had much higher incidence rates of tinea pedis and/or tinea unguium (Fig1). Also they tended to have both tinea pedis and tinea unguium (Fig 2). The incidence rates of tinea pedis and tinea unguium with dementia tended to be higher than those of healthy people of same ages. Patients with dementia might not to be able to perform daily foot care and maintain the proper hygiene for reasons of apathy or lack of interest. Those are the reasons why the patients with dementia could worsen their foot condition and get to have tinea pedis and tinea unguium. Our study suggests that dementia diseases, like diabetes mellitus and HIV infections, should be regarded as a risk factor for tinea pedis and tinea unguium.

\begin{tabular}{|c|c|c|c|}
\hline \multicolumn{4}{|c|}{ Table 1. Severity of tinea pedis by stage and extent } \\
\hline & \multicolumn{3}{|c|}{ Extent of lesion } \\
\hline Stage & Localized & Moderate & Widespread \\
\hline Early & 1 & 2 & 3 \\
\hline Intermediate & 2 & 3 & 4 \\
\hline Late & 4 & 5 & 6 \\
\hline
\end{tabular}

\begin{tabular}{|c|c|c|c|}
\hline Table 2 & $\begin{array}{c}\text { Total number of } \\
\text { patients }\end{array}$ & male & female \\
\hline & $\mathrm{N}=64$ & $\mathrm{~N}=25$ & $\mathrm{~N}=39$ \\
\hline Average age & $76.8 \pm 6.9$ & $74.7 \pm 6.9$ & $78.1 \pm 6.6$ \\
\hline Microscopic positive & $39 / 64(60.9 \%)$ & $15 / 25(60.0 \%)$ & $24 / 39(61.5 \%)$ \\
\hline Tinea pedis and tinea unguium & $20 / 39(51.3 \%)$ & $9 / 15(60.0 \%)$ & $11 / 24(45.8 \%)$ \\
\hline Tinea pedis only & $14 / 39(35.9 \%)$ & $6 / 15(40.0 \%)$ & $8 / 24(33.3 \%)$ \\
\hline Tinea unguium only & $5 / 39(12.8 \%)$ & $0 / 15(0.0 \%)$ & $5 / 24(20.8 \%)$ \\
\hline Severity of tinea pedis & $3.9 \pm 1.3$ & $3.9 \pm 1.2$ & $3.8 \pm 1.4$ \\
\hline cuiture positive & & & \\
\hline T. rubrum & $19 / 39(48.7 \%)$ & $6 / 15(40.0 \%)$ & $13 / 24(54.2 \%)$ \\
\hline T. mentagrophytes & $3 / 39(7.7 \%)$ & $1 / 15(6.7 \%)$ & $2 / 24(8.3 \%)$ \\
\hline
\end{tabular}

Fig2. Age distribution numbers of type of dermatophytes

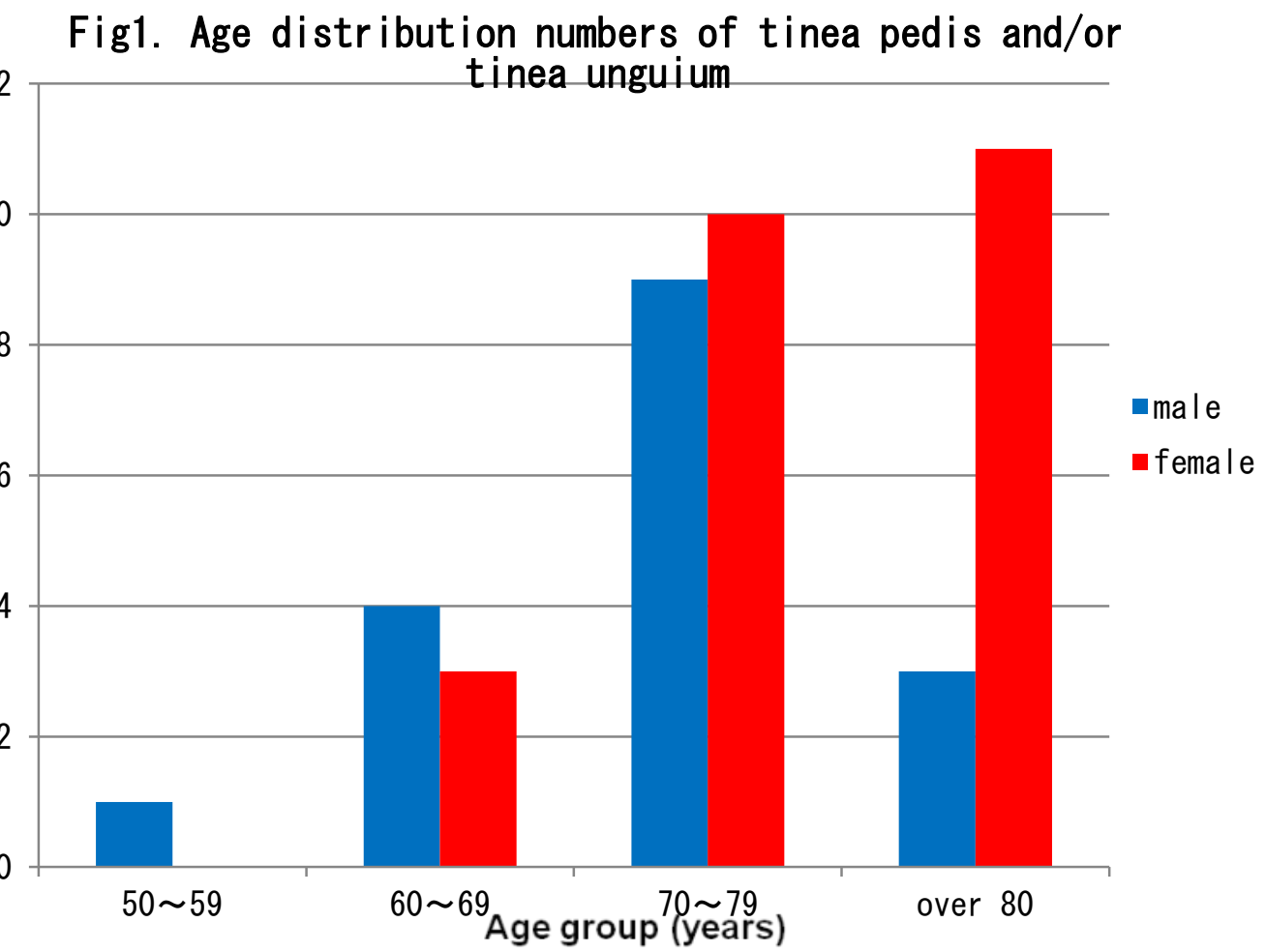

